\title{
WILEY-VCH
}

\section{Complex Morphogenesis by a Model Intrinsically Disordered Protein}

Constancio González-Obeso, Miguel González-Pérez, João F. Mano, Matilde Alonso, and José Carlos Rodríguez-Cabello*

Dr. C. González-Obeso, M. González-Pérez, Prof. M. Alonso, and Prof. J. C. RodríguezCabello

BIOFORGE (Group for Advanced Materials and Nanobiotechnology), University of Valladolid-CIBER-BBN, Paseo de Belén 19, 47011 Valladolid, Spain.

E-mail: roca@bioforge.uva.es

Dr. C. González-Obeso

Department of Biomedical Engineering, Tufts University, 4 Colby St., 02155 Medford, MA, USA.

Prof. J. F. Mano

Department of Chemistry, CICECO - Aveiro Institute of Materials, University of Aveiro, 3810-193 Aveiro, Portugal.

Keywords: self-assembly, silk-elastin-like recombinamers, biomorphs, phase transitions

\section{Abstract}

The development of intricate and complex self-assembling structures in the micrometer range, such as biomorphs, is a major challenge in materials science. Although complex structures can be obtained from self-assembling materials as they segregate from solution, their size is usually in the nanometer range or requires accessory techniques. Previous studies with intrinsically disordered proteins (IDPs) have shown that the active interplay of different molecular interactions provides access to new and more complex nanostructures. As such, we hypothesized that enriching the variety of intra- and intermolecular interactions in a model IDP will widen the landscape of sophisticated intermediate structures that can be accessed. In this study, we have built a model silk-elastin-like recombinamer (SELR) capable of interacting via three non-covalent interactions, namely hydrophobic, ion-pairing and H-bonding. This model material is shown to self-assemble into complex stable micrometer-sized biomorphs. Variation of the block composition, $\mathrm{pH}$ and temperature demonstrates the necessary interplay of all three interactions for the formation of such complex structures. 


\section{WILEY-VCH}

\section{Introduction}

The development of controllable, new self-assembling complex structures using naturally inspired biopolymers is of great interest nowadays. ${ }^{[1]}$ In Nature, the biosynthesis of protein-based networks, such as vertebrate elastic fibers, ${ }^{[2]}$ resilin $^{[3]}$ and silk, ${ }^{[4]}$ involves a phase transition from a solvated to a coacervate state. During this desolvation process, natural macromolecules evolve from a soluble unidimensional disordered chain of amino acids into a stable coacervate separated from the aqueous phase via a series of uncharacterized metastable conformations. ${ }^{[5]}$ The crosstalk of intermolecular interactions determines the equilibrium of the transient conformations, ${ }^{[6]}$ or stable secondary structures, ${ }^{[7]}$ that are formed. The final conformation on a nano- and microscale, ${ }^{[8]}$ as well as the final properties, are a result of these specific molecular arrangements and their evolution. The interacting regions in proteins are flanked by intrinsically disordered protein (IDP) regions, ${ }^{[9]}$ which provide them with conformational freedom to accommodate intermolecular interactions over time. The intrinsic disorder of these regions does not imply, however, that they are unable to actively participate in the interactions themselves. ${ }^{[10]}$ Final molecular arrangements correspond, sometimes, to complex far-from-equilibrium states stabilized by kinetic barriers that entrap these transient intermediates, ${ }^{[1]}$ ceasing their evolution towards thermodynamically more-favored states. ${ }^{[12]}$

In this regard, the family of elastin-like recombinamers (ELRs) ${ }^{[13]}$ may be of great help for gaining an insight into this phenomenon. The hydrophobic backbone of ELRs, which contains a tandem repeat of the (VPGXG) pentapeptide found in the intrinsically disordered regions of tropoelastin, ${ }^{[14]}$ confers a reversible lower critical solution temperature (LCST) on these systems ${ }^{[15]}$ which is controlled (among other factors) by the guest amino acid " $\mathrm{X}$ ". ${ }^{[16]}$ The thermodynamically stable state of ELRs is referred to as a statistical coil, ${ }^{[17]}$ in which only local and fluctuating secondary structures, such as 


\section{WILEY-VCH}

$\beta$-turns, can be detected. ${ }^{[18]}$ Molecular dynamics simulations have also shown that the backbone in ELRs remains highly disordered in the phase-separation stage ${ }^{[19]}$ and is responsible for the flexibility found in the native fibrils ${ }^{[20]}$ which, along with the lowenergy profile of the hydrophobic forces, generates a transient equilibrium between conformations with similar energies. ${ }^{[21]}$

The recombinant origin of ELRs permits to easily modify molecular features for changing and, ideally, controlling the coacervation pathway. For example, while amphiphilic block ELRs that exclusively coacervate via hydrophobic interactions have been shown to organize into nanometric spherical micelles ${ }^{[22]}$ and vesicles, ${ }^{[23]}$ the introduction of silk $\beta$-sheet-forming domains drives the formation towards amyloid fibers ${ }^{[24]}$ over time. Although the new structures are far from complex, this observation indicates an active crosstalk between interactions as, initially, the hydrophobic interactions trigger the formation of spheroid structures, whereas the silk $\beta$-sheet assembly, with its strong interactions and its particular kinetics, progressively stabilizes the transient conformations ${ }^{[25]}$ trapping new intermediate states. ${ }^{[26]}$ Even these relatively simple molecular events can have a strong influence on the micro and macrostructure of the final material. For example, certain silk-ELRs spontaneously form stable cellular hydrogels upon freezing an intermediate state between solution and full coacervate, without the use of any foaming agent. ${ }^{[27]}$ In these cases, silk motifs can be seen as both; a rigid and structured motif that opens new energy landscapes, but also as an "evolutionfreezer" motif. Other rigid structures have been combined with ELRs that lead to complex structures stabilized by the inclusion of unnatural amino acids and UV irradiation. $^{[28]}$

The exclusive presence of two different intermolecular interactions seems to be insufficient to provide a scenario in which multiple and complex molecular arrangements, such as those displayed by native proteins, can take place. Thus, a larger 


\section{WILEY-VCH}

pool of interactions seems to be necessary to access the complex structures found in vivo. ${ }^{[29]}$ To test this hypothesis, a SELR, referred to as EI-silk-VKV, which displays three different non-covalent forces, i.e. hydrophobic and electrostatic interactions, combined with hydrogen-bonded silk $\beta$-sheet domain, was designed. The hydrophobic interactions are responsible for the LCST of the material and trigger its phase transition upon a change in temperature, shaping the desolvated structures in the initial stages. Charge complementarity, a strong interaction that enables the formation of tight complexes between $\operatorname{IDPs}^{[30]}$, and the silk H-bonding are thought to shape the final structure. Silk H-bonding introduces both a tendency to form fibrillar structures and a kinetic barrier, thereby potentially arresting the segregation pathway in one of the transient intermediate states. The synergetic effect of these two competing interactions could then develop novel structures that exceed the complexity and size of the structures obtained to date.

In good agreement with our hypothesis, our results show the formation of colporate spherical particles in the micrometer range. The resemblance of the achieved assemblies to morphologies displayed by naturally occurring organic entities leads us to identify them as biomorphs. Moreover, selective suppression of the interplaying interactions demonstrates that crosstalk between the three interactions is essential to achieve these structures, while time and concentration dependence reveals information about their formation pathway.

\section{Results and discussion}

Figure S1 and Figure 1A show the hydrated and freeze-dried biomorph structures obtained upon incubation of EI-silk-VKV at $2 \mathrm{mg} \mathrm{mL}^{-1} 37^{\circ} \mathrm{C}$ for 48 hours, respectively. Under these conditions, all three non-covalent interactions participate, which gives rise to the complexity of the self-assembled structures. Incubation time (48 hours) was 


\section{WILEY-VCH}

sufficient time to allow the system to evolve towards a stable biomorph structure, as silk crosslinks were formed (Figure S2), trapping the evolution of the system by creating an energetic barrier. ${ }^{[12]}$

The EI-silk-VKV formed colporate biomorphic spheres surrounded and interconnected by filaments (Figure 1A). The observed morphologies mimic textures and patterns found in structures such as plant seeds, ${ }^{[31]}$ plankton $^{[32]}$ and pollen grains ${ }^{[33]}$ (Figure S3), thus prompting us to classify them as biomorphs. ${ }^{[34]}$ Although biomorphs found in the literature were originally obtained from pure inorganic systems ${ }^{[35]}$ bioinspired organic materials have emerged as powerful tools for fabricating organized architectures, ${ }^{[36]}$ thus demonstrating their potential for programming the self-assembly of biomaterial-based structures. A closer look at the colporate biomorphic particles reveals a radial core-shell structure, with a dense arrangement of fibers within the nucleus and a compact porous shell (Figure 1A, inset). The fibrous interconnected mesh that forms the core results in a random and small pore distribution, while the radially disposed planar structures of the shell form deep and non-interconnected pores on the surface. A population of disorganized fibers was also found surrounding the biomorphs.

In order to assess the effect of the different combinations of non-covalent interactions on the final structures, a series of experiments varying block composition, $\mathrm{pH}$ and temperature were carried out. Complementary studies, varying time and concentration, were also performed. Three recombinamers, namely SI-silk-VKV, EI-silk and EI (Figure 2), all lacking electrostatic interactions and, the latter, lacking the H-bonding silk motifs, were bioproduced and characterized (See Table TS3-4, Figure S4-7) for use as controls. The SIsilk-VKV developed a disorganized network of fibers accompanied by a small number of planar and globular structures Figure 1B. The tendency of SI-silk-VKV to form fibers, is thought to arise as a consequence of the silk $\beta$-sheet fibrillar nature, whereas planar structures are thought to arise from the coalescence of neighboring fibers. The EI-silk self-assembled into small, 


\section{WILEY-VCH}

irregular and randomly distributed planar structures (Figure 1C), although some flattened spheroid-like structures can also be observed. For the EI construct, a large number of small spheroids attached to planar structures was found (Figure 1D).

The EI construct is known to form spherical, self-assembled structures of different sizes, ${ }^{[22,37]}$ while large planar structures have been obtained once in combination with complementary peptide amphiphiles. ${ }^{[38]}$ Previous studies have shown that, with increasing concentration, and when allowed to self-assemble for short times (compared to our study), the EI di-block recombinamer self-assembles into filamentous interconnected structures formed by the coalescence of spherical aggregates. ${ }^{[39]}$ It therefore seems reasonable to suppose that allowing it to self-assemble for longer times could allow the interconnected filamentous structures to evolve into planar structures as a result of the balance between hydrophobic folding of the I block and the charged (i.e. hydrophilic) character of the E block. Such behavior is not as marked for EI-silk, as the presence of H-bonds may trap its evolution, leading to smaller surfaces. As can be seen, varying the polypeptide domains highlights the role played by the different non-covalent interactions and their effect on self-assembly at the micro scale, suggesting, as hypothetized, the necessary interplay of all three non-covalent interactions.

We further tested this conclusion by suppressing the electrostatic forces in the EI-silkVKV by modifying solution's $\mathrm{pH}$ below and above the pKa of glutamic acid/lysine side chain (namely pH 2 and 13), changing their negative/positive charged state to uncharged. Elimination of the electrostatic forces (as for the SI-silk-VKV, EI-silk and EI construct) did not result in the formation of biomorph-like assemblies. At $\mathrm{pH}$ 2, EI-silk-VKV selfassembled into filamentary and planar assemblies (Figure S8A). The first, thought to arise from the effect of silk, ${ }^{[40]}$ and the second, due to coalescing fibers, favored by the increased hydrophobicity of the construct (the E and I blocks are no longer water soluble). For the SI-silk-VKV control at $\mathrm{pH}$ 2, substitution of the hydrophobic E block 


\section{WILEY-VCH}

in favor of the $\mathrm{pH}$-insensitive $\mathrm{S}$ block alters the hydrophilicity distribution in this ELR, and thus its morphogenic pathway (Figure S8B) developing a different structure, characterized by small, irregular and interconnected surfaces. At pH 2, EI-silk and EI formed similar structures (Figure S8C-D), mainly planar and globular structures and short interconnected fibers (or proto-planar structures that have not evolved). Spherical structures are less abundant than in the case of EI-silk. The H-bonds of the silk motif could permit a further integration of the spheroids into the planar structures ${ }^{[40]}$. At $\mathrm{pH}$ 13, EI-silk-VKV formed granular structures that clumped together with no defined structure (Figure S9A). This amorphous morphology is thought to be related to the addition of $\mathrm{NaOH}$, which is responsible for the high $\mathrm{pH}$ and ionic strength, both of which hinder silk H-bonding. ${ }^{[41]}$ As result, the self-assembly process is governed only by hydrophobic interactions, also weakened by the high ionic strength, thereby impeding coalescence. ${ }^{[42]}$ Also result of this high $\mathrm{pH}$ and ionic strength, similar structures were observed for SI-silk-VKV, EI-silk and the EI controls (Figure S9B-D).

To demonstrate our effective control over the charged or uncharged state of the elastinlike blocks (Figure 2) by changing the $\mathrm{pH}$, and therefore over the presence of electrostatic interactions in the EI-silk-VKV, surface charge (Z-Potential) was measured as a function of $\mathrm{pH}$ and temperature. At $\mathrm{pH} 7$, when EI-silk-VKV is a zwitterionic molecule, and below the LCST, a small negative Z-Potential was found (Figure 3A), which decreased as temperature was increased above the LCST $(p \leq 0.05)$ (Figure 3B). This result confirms the stronger polarity/hydrophilicity of the glutamic acid elastin-like block compared to the lysine block, ${ }^{[16]}$ which remains exposed and shields the positive charge in the interior of the self-assembled structures. At $\mathrm{pH} 2$, and below the LCST, a slightly positive Z-Potential was found, with this value increasing further as the temperature increased above the LCST $(p<0.001)$ (Figure 3B). The uncharged E block acquires a preferred arrangement towards the interior of the assembled structures, 


\section{WILEY-VCH}

leaving the positively charged VKV block exposed on the surface. Negative Z-potential values, corresponding to the neutral form of the VKV block and exposure of the negatively charged E block, were found at $\mathrm{pH} 13$ both below and above the LCST ( $p \leq$ 0.05) (Figure 3B). The statistical analysis performed for the selected time points at the three $\mathrm{pH}$ values showed the consistent change of charge below and above the LCST (Figure 3B). It is worth noting that, regarding the evolution of the Z-potential at $\mathrm{pH} 7$, the first time point can be considered to be a discordant value, probably due to an artifact. With regard to the controls, at pH 7 SI-silk-VKV showed a positive charge above and below the LCST, being more positive above the $\operatorname{LCST}\left(\approx 0^{+}\right.$to $\left.+16 \mathrm{mV}, p<0.001\right)$ (Figure S10A), thus indicating reorganization of the SI-silk-VKV molecules to leave the VKV elastin block exposed on the surface. At $\mathrm{pH} 2$, and for all temperatures studied, SI-silk-VKV displayed a slightly positive Z-Potential $(\approx+2 \mathrm{mV})$, while at $\mathrm{pH} 13$, the ZPotential values were found to be slightly negative $(\approx-8 \mathrm{mV})$ (Figure S10A).

Similar behaviors were found at all $\mathrm{pH}$ values and temperatures for the EI-silk and EI constructs. At $\mathrm{pH} 7$, the Z-Potential values were found to be around $-24 \mathrm{mV}$ for all temperatures studied (Figure S10B-C). At pH 2 and at low temperature, the Z-Potential became slightly positive for both constructs, exhibiting a very similar behavior with temperature, whereas negative values centered at around $-8 \mathrm{mV}$ were found at $\mathrm{pH} 13$ (Figure S10B-C). The Z-Potential was of the expected sign for all constructs at both extreme $\mathrm{pH}$ values, but lower in magnitude. This finding can be explained by the shielding effect of the $\mathrm{HCl}$ and $\mathrm{NaOH}$ derived ions used to adjust the $\mathrm{pH} .{ }^{[43]}$ A statistical analysis demonstrated the consistency of the values found for each construct and under each condition. Block composition has a marked effect on the surface charge at $\mathrm{pH} 7$ (Figure S11A), whereas these effects are no longer statistically significant at pH 2 and 13 (Figure S11B-C). These results demonstrate that $\mathrm{pH}$ can be used to control the charges present in the ELRs, and thus the electrostatic interactions. ${ }^{[44]}$ 


\section{WILEY-VCH}

We then explored the effect of hydrophobic interactions on the formation of these biomorphic colporate particles. Hydrophobic forces were blocked by maintaining the solution temperature below the LCST of the elastin-like sequences, while electrostatic forces were regulated by means of $\mathrm{pH}$. A random mix of fibrillar, globular and planar structures was obtained for EI-silk-VKV at $\mathrm{pH} 7$ (Figure $\mathbf{S 1 2 A}$ ). These structures resembled those obtained with the same recombinamer at $37^{\circ} \mathrm{C}$ and $\mathrm{pH} 2$ (Figure S8A) and those obtained with SI-silk-VKV at $37^{\circ} \mathrm{C}$ and $\mathrm{pH} 7$ (Figure 1B), where only two out of three interactions are present (H-bonds+hydrophobic / H-bonds+electrostatic). Switching the solution $\mathrm{pH}$ to 2 hindered the electrostatic forces, thus leaving only the silk association via $\mathrm{H}$-bonds. The formation and progressive fusion of silk $\beta$-sheet assemblies via H-bonds resulted in a compact and continuous structure (Figure S12B). At $\mathrm{pH} 13$, hindered silk H-bonds, ${ }^{[41]}$ and the high ionic strength impaired the coalescence events thereby resulting in unstructured granular conglomerates (Figure S12C).

This set of experiments, in which the non-covalent forces were systematically hindered (by means of $\mathrm{pH}$ and/or temperature) or eliminated (by changing the block composition), showed that the synergetic interplay between the three interactions in combination with the intrinsically disordered character of these model proteins ${ }^{[21 b, 45]}$ is essential for formation of the colporate biomorph particles.

In order to better understand the EI-silk-VKV biomorph formation pathway, experiments varying time and concentration were performed. After 5 minutes at $37^{\circ} \mathrm{C}$ (Figure S13A), a mesh of planar and interconnected fibers in combination with a numerous and partially fused spheroids was found. After 1 hour, similar structures but with a smaller pore size between adjacent fibers dominated the self-assembly process (Figure S13B). Lateral growth as a result of the incorporation of material still held in solution/suspension may be responsible for the proto-planar surfaces observed. After 3 hours, the number of planar structures increased in at expenses of the number of fibers 


\section{WILEY-VCH}

and spheroids (Figure S13C). Longer incubation times (6,9 and 12 hours) allowed these proto-planar structures to evolve, closing the gaps between fibers and leading to the formation of small, interconnected flat surfaces (Figure S13D-F). After 24 hours (Figure S13G), planar structures combine to form closed, spiculated star-like "pockets" from which un-fused fibers emerge. Some spheroid structures can still be found, presumably arising from the small amount of material still held in solution/suspension. Longer times (36 hours Figure S13H) allow these structures to increase in size and number, while the number of fibers and spheroids found decreases. Finally, after incubation for 48 hours, colporate biomorph particles can be seen (Figure 1A).

To gain more information about this biomorphic assembly, the effect of concentration was explored. At the lowest concentration tested, namely $0.125 \mathrm{mg} \mathrm{mL}^{-1}$, nano-fibrillar and planar assemblies were found (Figure S14A). Even at this low concentration, the connected nature of these assemblies, with planar structures arising from neighboring and fused fibers, can be seen. Doubling the concentration increased the population of planar interconnected fibers, inducing the formation of larger (micrometer-sized) planar surfaces (Figure S14B). A further increase in concentration $\left(0.5 \mathrm{mg} \mathrm{mL}^{-1}\right)$ resulted in a dense and irregular pattern of fibers and surfaces (Figure S14C). At a concentration of $1 \mathrm{mg} \mathrm{mL} \mathrm{m}^{-1}$ (Figure S14D), randomly oriented planar structures and proto-colporate particles resembling the biomorphs start to appear. These proto-biomorph structures seem to arise from the fusion of the small planar structures formed. Some unfused fibers emerging from these proto-biomorph structures can be still found. Whereas, at $4 \mathrm{mg} \mathrm{mL}^{-}$ ${ }^{1}$, an intricate dense network of thick and irregular fibers was found (Figure S14E). These results demonstrate that, first, $2 \mathrm{mg} \mathrm{mL}^{-1}$ is the optimum concentration for the formation of the biomorphs and, second, that the dynamic and flexible system ${ }^{[8]}$ needs of sufficient time to accommodate all participating interactions to enrich and kinetically 


\section{WILEY-VCH}

trap the complex transient structures arising during the coacervation stage into the final biomorphs.

Turbidity and DLS permitted to assess the different assemblies formed by the EI-silkVKV and the controls under different conditions. The turbidity measurement for EI-silkVKV at $\mathrm{pH} 7$ showed that, as the temperature exceeds the LCST, the absorbance increased rapidly up to a maximum value (Figure 4A), and decreased with time due to the formation of large complexes that flocculate and deposit in the bottom of the cuvette. ${ }^{[46]}$ The marked increase in turbidity exhibited by EI-silk-VKV at pH 7 was not seen for any of the controls under the same conditions (Figure 4B-D), result of the formation of stable suspended structures ${ }^{[47]}$ and suggesting that the presence of electrostatic forces, in combination with the accompanying hydrophobic and H-bonding forces, triggers the self-assembly of EI-silk-VKV into larger aggregates than the other ELRs.

We then studied the pH scenarios in which the zwitterionic state of EI-silk-VKV (i.e. charge complementarity) is suppressed (pH 2 and 13). At $\mathrm{pH} 2$, the absorbance showed a sigmoidal increase, reaching a plateau value (Figure 4A) similar to that for the controls at neutral pH. SI-silk-VKV, which is insensitive to low $\mathrm{pH}$, behaved the same as at neutral $\mathrm{pH}$ (Figure 4B). For those controls bearing the E block, the turbidity exhibited a sharp increase as the temperature increased above the LCST (Figure 4C-D), reaching values similar to those found for EI-silk-VKV at $\mathrm{pH} 7$ (Figure 4A), subsequently decreasing over time. As the temperature increases, both the E and I blocks lose their water-solubility, thus leading to coacervation of the recombinamers into large aggregates that flocculate and precipitate over time, thus increasing the amount of recombinamer at the bottom of the cuvette and decreasing the turbidity (Figure 4C-D). ${ }^{[46 b]}$ This behavior was not observed for the VKV-containing SELRs as this block remains charged and water-soluble, thus preventing them from aggregating and precipitating (Figure $4 \mathbf{A}$ - 


\section{WILEY-VCH}

B). ${ }^{[47]}$ It is worth noting that, at $\mathrm{pH}$ 2, all E block-containing recombinamers exhibited a double shift in turbidity, which was associated with the phase transition of the neutral E and I elastin-like blocks, as demonstrated in the SI (Figure S15-16). With regard to pH 13, no significant differences were found between EI-silk-VKV and the controls (Figure 4A-D). A single transition associated with the I block led to a small increase in absorbance that was maintained over time, thus indicating the formation of stable particles held in solution by the hydrophilic E or S block.

DLS, a complementary technique to turbidity, was used to measure changes in the volume of the self-assembled structures with temperature and time. For EI-silk-VKV at pH 7, and below the LCST, nanoscale aggregates which might have been formed during the freeze-drying process ${ }^{[48]}$ or may arise from the electrostatic forces, were found (Figure S17A). Silk H-bonding crosslinking would have a small effect due to the reduced time available for the secondary structures to form. ${ }^{[24 a, 49]}$ Once above the LCST of the I block, an increase followed by a decrease in particle volume was observed (Figure S17A). For the controls, temperatures above the LCST led to a slight increase in volume, which remained stable over time (Figure S17B-D). This result was replicated by EI-silk-VKV at pH 2 and 13, where the zwitterionic state is missing, and SI-silkVKV at all three $\mathrm{pH}$ values (Figure S17). At pH 2 above the LCST, the EI-silk and EI controls formed large hydrophobic aggregates (Figure S17C-D) and flocculated, replicating the sharp increase and subsequent decrease observed in absorbance (Figure 4C-D). The evolution of volume with temperature, at different $\mathrm{pH}$ (Figure S17), was found to be similar to that observed by turbidimetry (Figure $4 \mathbf{A - D}$ ), supporting the consistency of the results and the importance of the interplaying non-covalent interactions in the formation of the EI-silk-VKV micrometer-sized structures, while for the controls, lacking electrostatic interactions, to stable nanometer-sized aggregates or flocculating hydrophobic clusters. 


\section{WILEY-VCH}

A time-lapse of the normalized size distribution allowed us to observe the evolution of the self-assembled structures with temperature, $\mathrm{pH}$ and time (Figure 4E-P). For EI-silk$\mathrm{VKV}$, at $\mathrm{pH} 7$ and below the LCST, a polydisperse family of aggregated structures was found (Figure 4E). These aggregates are related to the formation of silk nucleation points derived from the freeze-drying step ${ }^{[48]}$ (also present at $\mathrm{pH} 2$ and 13) being the electrostatic forces responsible of the larger aggregates found at $\mathrm{pH} 7$ but not at $\mathrm{pH} 2$ and 13. As temperature increases above the LCST, the aggregates evolve into a more monodisperse distribution (centered at $56.1 \mu \mathrm{m}$ ), although signals above the micrometer size, which result from the aggregation of material via electrostatic interactions, can be seen. Such structures grow rapidly by incorporating additional material via the three participating non-covalent forces that, with time, precipitate as seen by the absence of a signal after reaching $30^{\circ} \mathrm{C}$. This coalescence event ceased once the electrostatic interactions had been blocked at $\mathrm{pH} 2$ and $\mathrm{pH} 13$, resulting in a stable size distribution centered at around 58.8 and $32.7 \mathrm{~nm}$, respectively (Figure 4F-G). This decrease in size was related to the high ionic strength at $\mathrm{pH} 13$.

At neutral pH (Figure 4H), SI-silk-VKV followed a similar trend to that observed for EI-silk-VKV at $\mathrm{pH} 2$ and 13. At $\mathrm{pH} 2$, in the presence of identical interactions, this trend was preserved (Figure 4I), although a narrowing of the distribution was observed due to the added $\mathrm{HCl}^{[42]}$ At $\mathrm{pH}$ 13, hindered H-bonds ${ }^{[41]}$ lead the assembly of the SI-silkVKV above the LCST to hydrophobic interactions, resulting in a broad single size distribution centered at $\approx 50.7 \mathrm{~nm}$ (Figure $4 J)$, slightly smaller than for the EI-silk-VKV, as substitution of the charged $\mathrm{E}$ block for the polar $\mathrm{S}$ block reduced the compacting effect of the high ionic strength. ${ }^{[42]}$ At $\mathrm{pH} 7$, EI-silk and EI differed in the number and proportion of distributions below the LCST (Figure 4K,N), finding distributions of similar intensity at 10,164, 712 and $5560 \mathrm{~nm}$ for the EI-silk, and a predominant distribution at $13 \mathrm{~nm}$ accompanied by 459 and $5560 \mathrm{~nm}$ aggregates for the EI. This was 


\section{WILEY-VCH}

attributed to the presence of silk motifs, absent in the EI. For both constructs, an increase in temperature and time resulted in the disappearance of the smaller distribution centered at $10 \mathrm{~nm}$ in favor of a preferred assembly centered at around $100 \mathrm{~nm}$. The limited formation of silk $\beta$-sheet structures at short times ${ }^{[24 a, 49]}$ suggests that the self-assembly of EI-silk occurs due to the hydrophobic forces, thereby mirroring the self-assembly of EI with time. At longer times, the size distribution of EI-silk narrows, presumably due to the tight interaction between silk motifs found in the core of the particle (as seen with Z-Potential, Figure S10B). At pH 2, and above the LCST, EI-silk and EI formed aggregates that grew in size above the measurable scale (Figure 4L,OO). The increased hydrophobic character of the aggregates in suspension induces a progressive aggregation and flocculation with time. For the EI-silk and EI controls, temperatures above the LCST at $\mathrm{pH} 13$ induced the appearance of a single distribution centered at $43.8 \mathrm{~nm}$ (Figure 4M,P). Interestingly, the size-distribution narrowing effect of the silk motifs in EI-silk is now not seen (Figure 4M), as silk interactions are hindered under these $\mathrm{pH}$ conditions,${ }^{[41]}$ remaining disordered, and thus forming bulkier sequences. ${ }^{[12]}$

The thermoresponsiveness of the recombinamers was confirmed by DSC. The transition of the I block was evident for all recombinamers at $\mathrm{pH} 7$ (Figure S18A-D), with the presence of a single peak indicating that the polar S and negatively and positively charged $\mathrm{E}$ and VKV blocks, respectively, remain solvated at this $\mathrm{pH}$. At $\mathrm{pH}$ 2, a second transition appeared for those constructs containing the E block (Figure S18A,C-D), whereas a single peak was found for SI-silk-VKV (Figure S18B). The side chain in glutamic acid is uncharged at this $\mathrm{pH}$, (as seen from the Z-Potential Figure S11B), thereby decreasing the polarity and LCST of the E block within the window of temperatures studied. Analysis of the Ex15 and Ix120 mono-blocks helped distinguish each transition at $\mathrm{pH} 2$. Thus, the sharper peak exhibited by Ix120 when compared to Ex15 (Figure S18E-F) allowed us to assign the first and second transitions to the I and 


\section{WILEY-VCH}

E blocks, respectively. This order, which contrasts with that observed by turbidity

(Figure S15-16), was attributed to the concentration gap between measurements, i.e. DSC $50 \mathrm{mg} \mathrm{mL}^{-1}$ and turbidity $1 \mathrm{mg} \mathrm{mL}^{-1}$. As has been described previously, varying the concentration produces a different shift in the transition temperature of the isoleucine and glutamic acid residues, thus switching the order found. ${ }^{[50]}$ At $\mathrm{pH} 13$, and despite the neutral form of the VKV block, a single I block transition was found in the DSC thermograms (Figure S18A-B). According to previous studies, ${ }^{[51]}$ the apolar I block could increase the hydrophobic hydration in the VKV block, thus shifting the LCST towards higher values. Moreover, the LCST and enthalpy values were found to be dependent on the polarity of the accompanying building blocks. Introduction of the silk motif, VKV block and further substitution of the $\mathrm{E}$ for the $\mathrm{S}$ block progressively increased the hydrophilicity of the construct, thereby increasing the LCST while decreasing the associated enthalpy (Figure S18G-H). ${ }^{[52]}$

\section{Conclusion}

We have designed an intrinsically disordered protein (IDP) containing three elastin-like blocks and a silk-like domain to investigate and exploit the effect of the hydrophobic, electrostatic, and H-bonding $\beta$-sheet structures on the morphogenesis that governs the phase transition of the protein from a soluble to an insoluble state. Selection of the participating forces by varying the $\mathrm{pH}$, temperature, time and block composition has shown our ability to modulate and arrest the dynamic self-assembly of the SELR chains, obtaining structures ranging from fibrillar and planar structures to colporate spheres, identified as biomorphs. The complexity of the biomorphs, only found when all three interactions are present, reveals the synergetic interplay between the encoded non-covalent interactions, which sculpt the intrinsically disordered and flexible polypeptides into complex intermediate states that become kinetically trapped as a result of the silk $\beta$-sheet domains. This work sheds light upon the supramolecular self-assembly 


\section{WILEY-VCH}

pathway by defining the spontaneous development of shape in proteinaceous biomolecules at various length scales while offering a promising strategy for the manufacture of novel and morphologically rich structures and shapes with potential biological functionalities, such as those found in nature, via a bottom-up approach. These biomimetic and tailorable SELR proteins represent a suitable platform for studying the independent or combined effect of different non-covalent interactions on the development, upon desolvation in time and space, of nano- to micormetric structures of interest in the materials science field.

\section{Acknowledgements}

The authors are grateful for the funding from the Spanish Government (MAT2016-78903-R, RTI2018-096320-B-C22, FPU15-00448), Junta de Castilla y León (VA317P18), Interreg V A España Portugal POCTEP (0624_2IQBIONEURO_6_E) and Centro en Red de Medicina Regenerativa y Terapia Celular de Castilla y León.

Received: ((will be filled in by the editorial staff))

Revised: ((will be filled in by the editorial staff)) Published online: ((will be filled in by the editorial staff))

\section{References}

[1] a) A. Majerle, D. T. Schmieden, R. Jerala, A. S. Meyer, Biochemistry 2019, 58, 2095;

b) B. O. Okesola, A. Mata, Chem. Soc. Rev. 2018, 47, 3721.

[2] R. P. Mecham, J. E. Heuser, Cell Biol. Extracell. Matrix 1991, 79.

[3] S. O. Andersen, T. Weis-Fogh, Adv. Insect Physiol. 1964, 1.

[4] Y. Shen, M. A. Johnson, D. C. Martin, Macromolecules 1998, 31, 8857.

[5] V. N. Uversky, Adv. Colloid Interface Sci. 2017, 239, 97.

[6] L. D. Muiznieks, A. S. Weiss, F. W. Keeley, Biochem. Cell Biol. 2010, 88, 239. 


\section{WILEY-VCH}

[7] S. A. Fossey, G. Némethy, K. D. Gibson, H. A. Scheraga, Biopolymers 1991, 31, 1529.

[8] J. R. Simon, N. J. Carroll, M. Rubinstein, A. Chilkoti, G. P. López, Nat. Chem. 2017, 9, 509.

[9] T. Wald, F. Spoutil, A. Osickova, M. Prochazkova, O. Benada, P. Kasparek, L. Bumba, O. D. Klein, R. Sedlacek, P. Sebo, J. Prochazka, R. Osicka, Proc. Natl. Acad. Sci. U.S.A. 2017, 201615334.

[10] W. F. Zeno, U. Baul, W. T. Snead, A. C. M. DeGroot, L. Wang, E. M. Lafer, D. Thirumalai, J. C. Stachowiak, Nat. Commun. 2018, 9, 4152.

[11] R. L. Baldwin, J. Biomol. NMR 1995, 5, 103.

[12] I. W. Hamley, V. Castelletto, Bioconj. Chem. 2017, 28, 731.

[13] J. C. Rodríguez-Cabello, L. Martín, M. Alonso, F. J. Arias, A. M. Testera, Polymer 2009, 50, 5159.

[14] B. Vrhovski, A. S. Weiss, Eur. J. Biochem. 1998, $258,1$.

[15] N. K. Li, F. G. Quiroz, C. K. Hall, A. Chilkoti, Y. G. Yingling, Biomacromolecules 2014, 15, 3522 .

[16] D. W. Urry, D. C. Gowda, T. M. Parker, C.-H. Luan, M. C. Reid, C. M. Harris, A. Pattanaik, R. D. Harris, Biopolymers 1992, 32, 1243.

[17] A. K. Jha, A. Colubri, K. F. Freed, T. R. Sosnick, Proc. Natl. Acad. Sci. U.S:A. 2005, $102,13099$.

[18] a) S. Rauscher, R. Pomès, eLife 2017, 6, e26526; b) A. M. Tamburro, B. Bochicchio, A. Pepe, Biochemistry 2003, 42, 13347.

[19] a) S. Rauscher, S. Baud, M. Miao, Fred W. Keeley, R. Pomès, Structure 2006, 14, 1667;

b) L. D. Muiznieks, F. W. Keeley, J. Biol. Chem. 2010, 285, 39779.

[20] H. Vindin, S. M. Mithieux, A. S. Weiss, Matrix Biol. 2019, 84, 4.

[21] a) L. D. Muiznieks, S. Sharpe, R. Pomès, F. W. Keeley, J. Mol. Biol. 2018, 430, 4741;

b) S. Roberts, M. Dzuricky, A. Chilkoti, FEBS Lett. 2015, 589, 2477. 


\section{WILEY-VCH}

[22] C. García-Arévalo, J. F. Bermejo-Martín, L. Rico, V. Iglesias, L. Martín, J. C. Rodríguez-Cabello, F. J. Arias, Mol. Pharm. 2013, 10, 586.

[23] L. Martín, E. Castro, A. Ribeiro, M. Alonso, J. C. Rodríguez-Cabello, Biomacromolecules 2012, 13, 293.

[24] a) A. Fernández-Colino, F. J. Arias, M. Alonso, J. C. Rodríguez-Cabello, Biomacromolecules 2014, 15, 3781; b) N. Varongchayakul, S. Johnson, T. Quabili, J. Cappello, H. Ghandehari, S. D. J. Solares, W. Hwang, J. Seog, ACS Nano 2013, 7, 7734.

[25] Q. Lu, H. Zhu, C. Zhang, F. Zhang, B. Zhang, D. L. Kaplan, Biomacromolecules 2012, 13,826 .

[26] a) X. Hu, Q. Lu, D. L. Kaplan, P. Cebe, Macromolecules 2009, 42, 2079; b) X. Chen, Z. Shao, N. S. Marinkovic, L. M. Miller, P. Zhou, M. R. Chance, Biophys. Chem. 2001, $89,25$.

[27] A. Ibáñez-Fonseca, D. Orbanic, F. J. Arias, M. Alonso, D. I. Zeugolis, J. C. RodríguezCabello, Small 2020, 2001244.

[28] S. Roberts, V. Miao, S. Costa, J. Simon, G. Kelly, T. Shah, S. Zauscher, A. Chilkoti, Nat. Commun. 2020, 11, 1342.

[29] a) H.-X. Zhou, X. Pang, Chem. Rev. 2018, 118, 1691; b) P. Aloy, R. B. Russell, Nat. Biotechnol. 2004, 22, 1317; c) H. Zheng, C. Lu, J. Lan, S. Fan, V. Nanda, F. Xu, Proc. Natl. Acad. Sci. U.S.A. 2018, 115, 6207.

[30] A. Borgia, M. B. Borgia, K. Bugge, V. M. Kissling, P. O. Heidarsson, C. B. Fernandes, A. Sottini, A. Soranno, K. J. Buholzer, D. Nettels, B. B. Kragelund, R. B. Best, B. Schuler, Nature 2018, 555, 61.

[31] L. B. Enrich, M. L. Scheuermann, A. Mohadjer, K. R. Matthias, C. F. Eller, M. S. Newman, M. Fujinaka, T. Poon, Tetrahedron Lett. 2008, 49, 2503.

[32] O. Roger Anderson, P. Bennett, Mar. Micropaleontol. 1985, 9, 441. 


\section{WILEY-VCH}

[33] a) H. Özler, S. Pehlivan, A. Kahraman, M. Doğan, F. Celep, B. Başer, A. Yavru, S. Bagherpour, Flora 2011, 206, 316; b) S. Srisuwan, D. Sihachakr, J. Martín, J. Vallès, A. Ressayre, S. Brown, S. Siljak-Yakovlev, Plant Biol. 2018, 21.

[34] J. M. GarcÍa-Ruiz, Origins Life Evol. B. 1994, 24, 451.

[35] J. M. García-Ruiz, S. T. Hyde, A. M. Carnerup, A. G. Christy, M. J. Van Kranendonk, N. J. Welham, Science 2003, 302, 1194.

[36] a) N. Sánchez-Puig, E. Guerra-Flores, F. López-Sánchez, P. A. Juárez-Espinoza, R. Ruiz-Arellano, R. González-Muñoz, R. Arreguín-Espinosa, A. Moreno, J. Mater. Sci. 2012, 47, 2943; b) S. Elsharkawy, M. Al-Jawad, M. F. Pantano, E. Tejeda-Montes, K. Mehta, H. Jamal, S. Agarwal, K. Shuturminska, A. Rice, N. V. Tarakina, R. M. Wilson, A. J. Bushby, M. Alonso, J. C. Rodriguez-Cabello, E. Barbieri, A. del Río Hernández, M. M. Stevens, N. M. Pugno, P. Anderson, A. Mata, Nat. Commun. 2018, 9, 2145.

[37] J.-W. Ro, H. Choi, T.-Y. Heo, S.-H. Choi, J.-I. Won, Biotechnol. Bioprocess Eng. 2018, 23,627 .

[38] K. E. Inostroza-Brito, E. Collin, O. Siton-Mendelson, K. H. Smith, A. Monge-Marcet, D. S. Ferreira, R. P. Rodríguez, M. Alonso, J. C. Rodríguez-Cabello, R. L. Reis, F. Sagués, L. Botto, R. Bitton, H. S. Azevedo, A. Mata, Nat. Chem. 2015, 7, 897.

[39] M. H. Misbah, L. Quintanilla, M. Alonso, J. C. Rodríguez-Cabello, Polymer 2015, 81, 37.

[40] D. Xu, C. Guo, G. P. Holland, Biomacromolecules 2015, 16, 2072.

[41] H. Sun, B. Marelli, Nat. Commun. 2020, 11, 351.

[42] W. Hwang, B.-H. Kim, R. Dandu, J. Cappello, H. Ghandehari, J. Seog, Langmuir 2009, $25,12682$.

[43] S. Salgin, U. Salgin, S. Bahadir, Int. J. Electrochem. Sci. 2012, 7, 12404.

[44] F. Franks, Protein Biotechnol. 1993, 489.

[45] K. M. Ruff, S. Roberts, A. Chilkoti, R. V. Pappu, J. Mol. Biol. 2018, 430, 4619. 


\section{WILEY-VCH}

[46] a) G. Pinedo-Martín, M. Santos, A. M. Testera, M. Alonso, J. C. Rodríguez-Cabello, Polymer 2014, 55, 5314; b) D. E. Meyer, K. Trabbic-Carlson, A. Chilkoti, Biotechnol. Prog. 2001, 17, 720 .

[47] M. R. Dreher, A. J. Simnick, K. Fischer, R. J. Smith, A. Patel, M. Schmidt, A. Chilkoti, J. Am. Chem. Soc. 2008, 130, 687.

[48] a) A. Girotti, D. Orbanic, A. Ibáñez-Fonseca, C. Gonzalez-Obeso, J. C. RodríguezCabello, Adv. Healthc. Mater. 2015, 4, 2423; b) M. Li, S. Lu, Z. Wu, H. Yan, J. Mo, L. Wang, J. Appl. Polym. Sci. 2001, 79, 2185.

[49] F. Cipriani, M. Krüger, I. G. de Torre, L. Q. Sierra, M. A. Rodrigo, L. Kock, J. C. Rodriguez-Cabello, Biomacromolecules 2018, 19, 4333.

[50] a) T. Yamaoka, T. Tamura, Y. Seto, T. Tada, S. Kunugi, D. A. Tirrell, Biomacromolecules 2003, 4, 1680; b) A. Girotti, J. Reguera, F. J. Arias, M. Alonso, A. M. Testera, J. C. Rodríguez-Cabello, Macromolecules 2004, 37, 3396.

[51] A. Ribeiro, F. J. Arias, J. Reguera, M. Alonso, J. C. Rodríguez-Cabello, Biophys. J. 2009, 97, 312 .

[52] J. Reguera, D. W. Urry, T. M. Parker, D. T. McPherson, J. C. Rodríguez-Cabello, Biomacromolecules 2007, 8, 354. 


\section{WILEY-VCH}

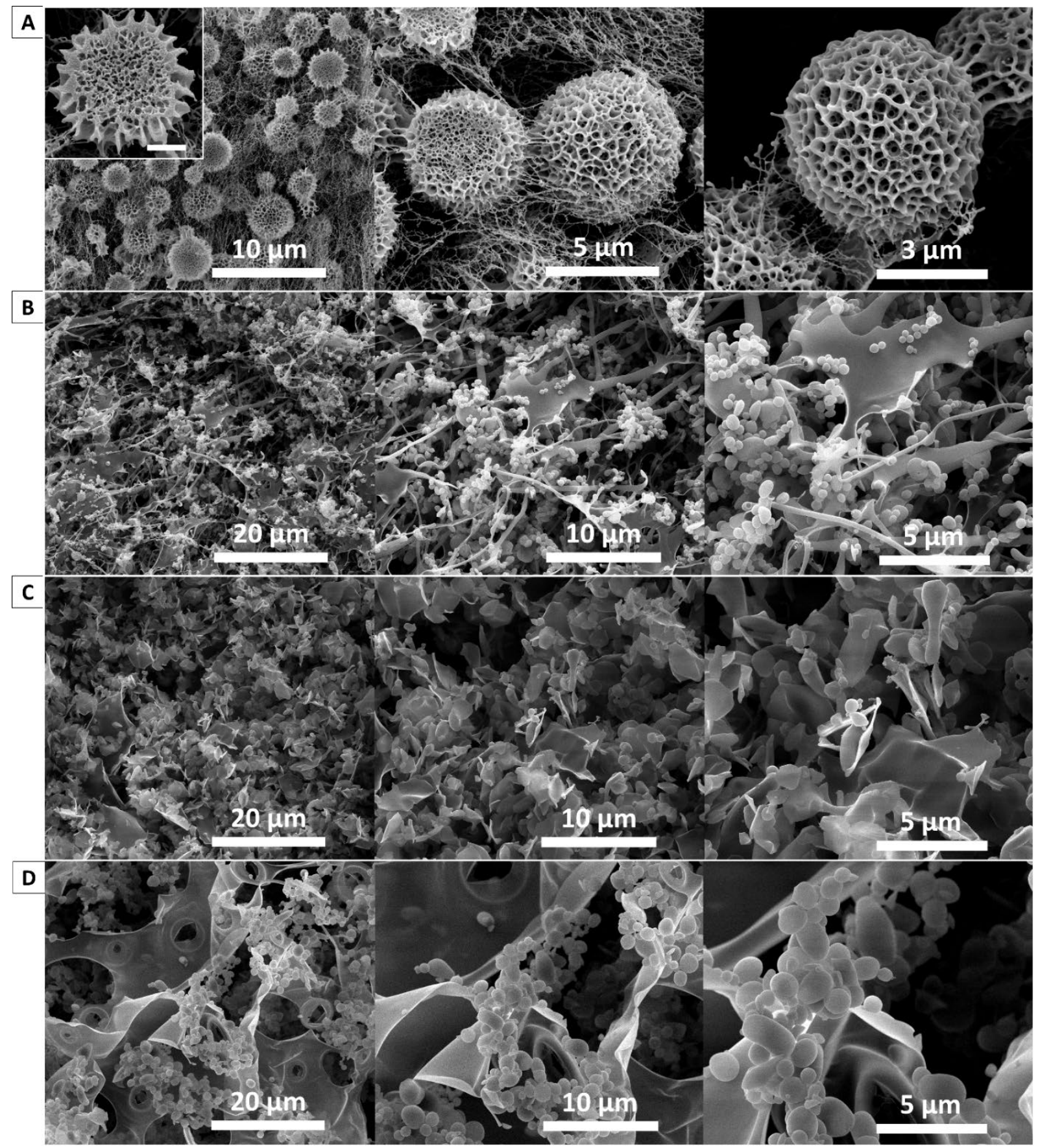

Figure 1 Representative SEM images of the self-assembled structures generated by (A) EI-silk$\mathrm{VKV}$, (B) SI-silk-VKV, (C) EI-silk and (D) EI from $2 \mathrm{mg} \mathrm{mL}^{-1}$ aqueous solutions at $\mathrm{pH} 7$ after 48 hours at $37^{\circ} \mathrm{C} .1 \mu \mathrm{m}$ scale bar in the inset of Figure $1 \mathrm{~A}$. 


\section{WILEY-VCH}

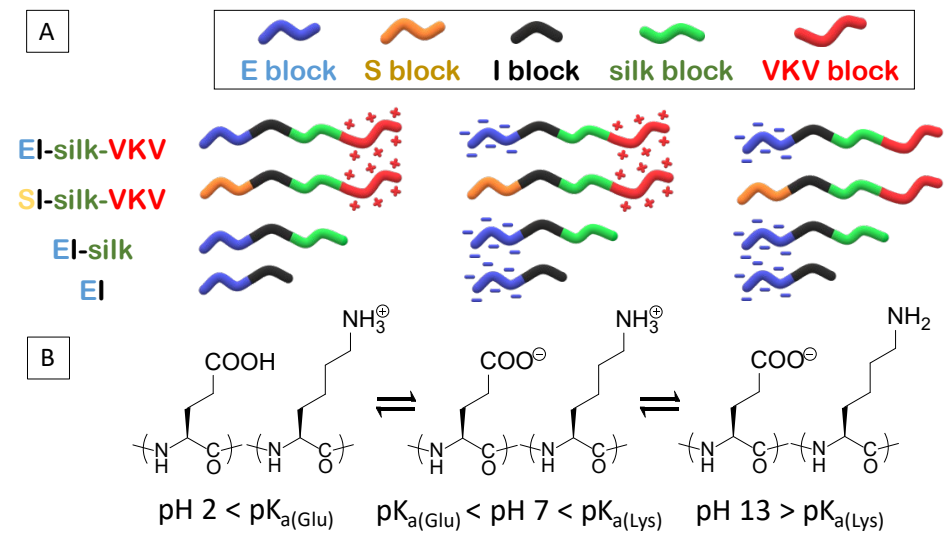

Figure 2 (A) Schematic representation of the EI-silk-VKV, SI-silk-VKV, EI-silk and EI elastinlike and silk domains and (B) glutamic acid and lysine's charge dependence with $\mathrm{pH}$.

\begin{tabular}{l|l|l|l|l}
$\mathrm{pH} 7$ & $\mathrm{pH} 2$ & $\mathrm{pH} 13$
\end{tabular}

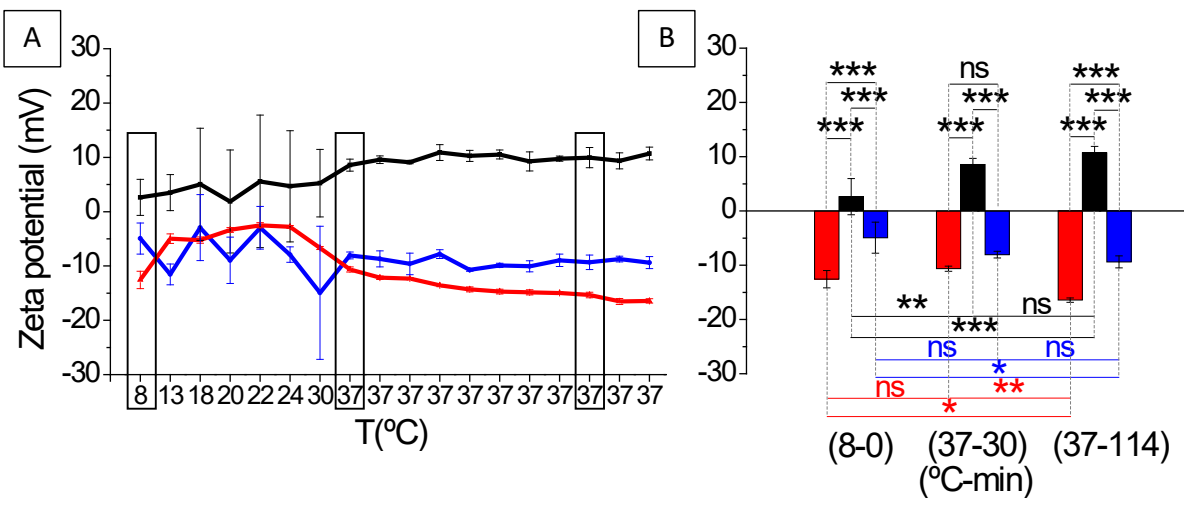

Figure 3 (A) Zeta potential measurements for EI-silk-VKV at $1 \mathrm{mg} \mathrm{mL}^{-1}$ in ultrapure water at pH 7 (red), 2 (black) and 13 (blue) accompanied by the standard deviation. (B) Statistical comparison between the zeta potential values for EI-silk-VKV at $8^{\circ} \mathrm{C}$, at the first and last time point at $37^{\circ} \mathrm{C}$ and $\mathrm{pH} 7,2$ and 13. $n s p>0.05,{ }^{*} p \leq 0.05,{ }^{*} p<0.01$ and ${ }^{* * *} p<0.001$. 


\section{WILEY-VCH}
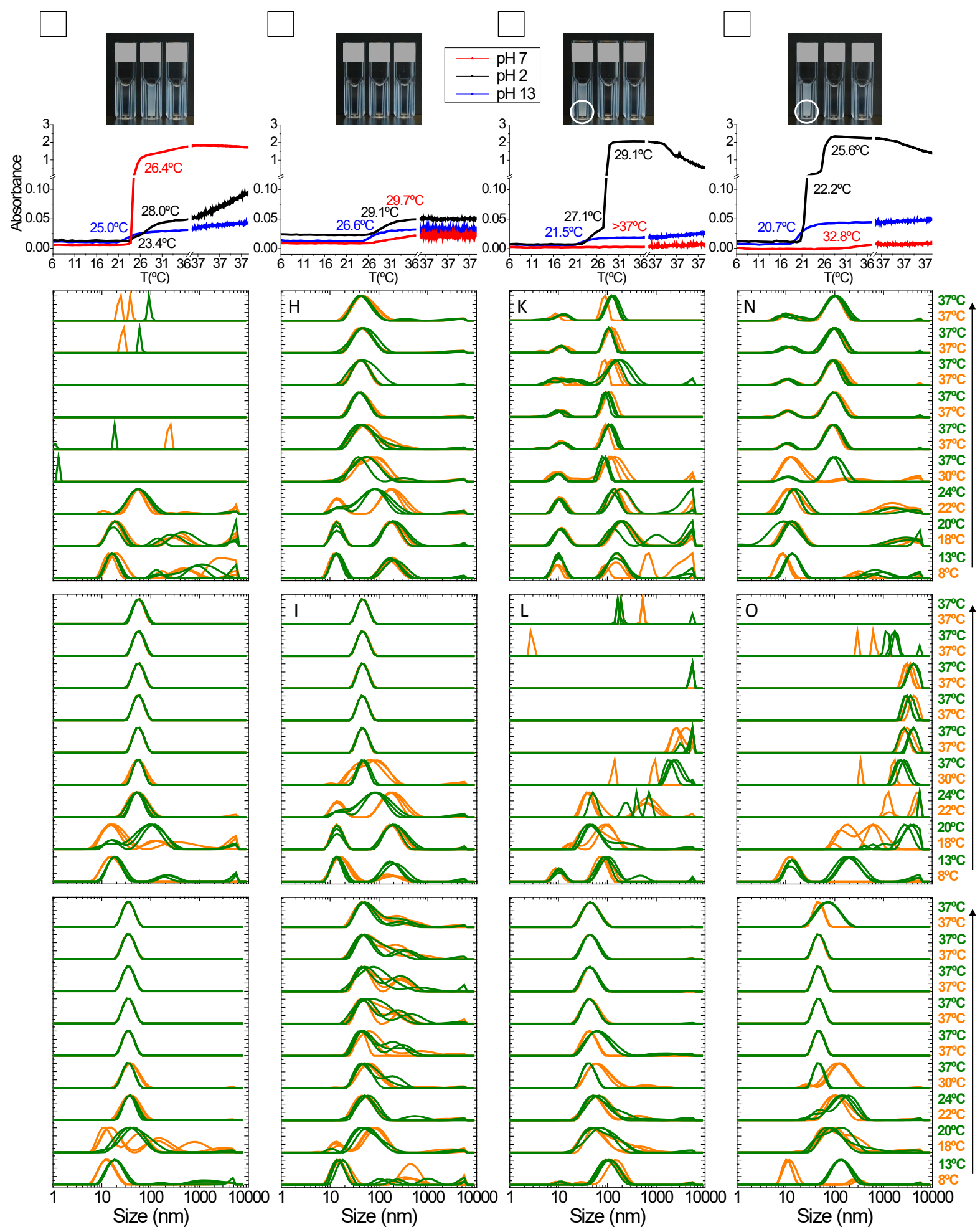

Figure 4 Turbidity curves for (A) EI-silk-VKV, (B) SI-silk-VKV, (C) EI-silk and (D) EI at $12.375 \mu \mathrm{M}$ in ultrapure water at $\mathrm{pH} 7$ (red), 2 (black) and 13 (blue) accompanied by representative images of the respective solutions heated to $37^{\circ} \mathrm{C}$ at $\mathrm{pH} 2,7$ and 13 , where a white circle surrounds the precipitating EI-silk and EI accumulated at the bottom of the cuvette at $\mathrm{pH}$ 2. Variation in normalized size distributions with temperature for (E-G) EI-silk-VKV, (H-J) SI-silk-VKV, (K-M) EI-silk and (N-P) EI at $1 \mathrm{mg} \mathrm{mL}^{-1}$ in ultrapure water at $\mathrm{pH} 7,2$, and 13. 


\section{WILEY-VCH}

\section{Table of contents}

Non-covalent forces modulate the phase transition of naturally occurring self-assembled structures. By varying the block composition, $\mathrm{pH}$ and temperature, it has been demonstrated the necessary synergetic combination of three non-covalent interactions present in an intrinsically disordered silk-elastin-like recombinamer for self-assembly into micrometersized complex biomorphs.

C. González-Obeso, M. González-Pérez, J. F. Mano, M. Alonso, J. C. Rodríguez-Cabello*

\section{Complex Morphogenesis by a Model Intrinsically Disordered Protein}

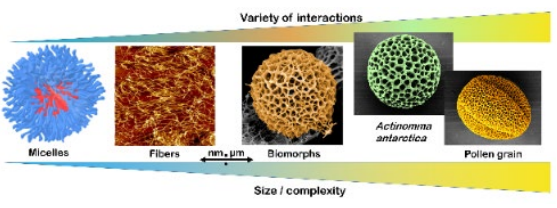

